(c) 2013 The Author(s)

This article is published with open access at Springerlink.com

\title{
Sharp Logarithmic Bounds for Beurling-Ahlfors Operator Restricted to the Class of Radial Functions
}

\author{
Adam Osȩkowski
}

\begin{abstract}
We establish a class of sharp logarithmic estimates for the Beurling-Ahlfors transform $B$ on the complex plane. For any $K>0$ we determine the optimal constant $L=L(K) \in(0, \infty]$ such that the following holds. If $F: \mathbb{C} \rightarrow \mathbb{C}$ is a radial function, then for any $R>0$,

$$
\frac{1}{|\mathcal{B}(0, R)|} \int_{\mathcal{B}(0, R)}|B F(z)| \mathrm{d} z \leq \frac{K}{|\mathcal{B}(0, R)|} \int_{\mathcal{B}(0, R)} \Psi(|F(z)|) \mathrm{d} z+L(K),
$$

where $\Psi(t)=(t+1) \log (t+1)-t$ and $\mathcal{B}(0, R) \subset \mathbb{C}$ denotes the ball of center 0 and radius $R$. A related result in higher dimensions is also established. The proof rests on probabilistic methods and exploits a certain sharp inequality for martingales.
\end{abstract}

Mathematics Subject Classification (2010). Primary 42B20; Secondary $60 \mathrm{G} 44$.

Keywords. Beurling-Ahlfors operator, martingale, radial function, LlogL inequality.

\section{Introduction}

The Beurling-Ahlfors transform is an operator acting on $L^{p}(\mathbb{C})$, defined by the singular integral

$$
B F(z)=-\frac{1}{\pi} \text { p.v. } \int_{\mathbb{C}} \frac{F(w)}{(z-w)^{2}} \mathrm{~d} w,
$$

where p.v. means the principal value and the integration is with respect to the Lebesgue measure on the plane. Alternatively, it can be defined as a Fourier multiplier with the symbol $m(\xi)=\bar{\xi} / \xi$, i.e., we have the identity $\widehat{B F}(\xi)=$ $\bar{\xi} \widehat{F}(\xi)$ for all $\xi \in \mathbb{C} \backslash\{0\}$. This operator is of fundamental importance in

Partially supported by Polish Ministry of Science and Higher Education (MNiSW) grant IP2011 039571 'Iuventus Plus'. 
the study of quasiconformal mappings and partial differential equations, see e.g. [2], [3], [17] and references therein. There is a challenging question about the precise value of the $L^{p}$ norms of this operator, the answer to which would imply several deep results related to the Gehring-Reich conjecture [12] on the area distortion of quasiconformal mapping in the plane (which has been proved by Astala in [1]). Motivated by this problem, T. Iwaniec [14] conjectured that

$$
\|B\|_{L^{p}(\mathbb{C}) \rightarrow L^{p}(\mathbb{C})}=p^{*}-1,
$$

where $p^{*}=\max \{p, p /(p-1)\}$, and the question of whether it is true or not is open for thirty years now. In fact, the main difficulty lies in proving the upper bound $\|B\|_{L^{p}(\mathbb{C}) \rightarrow L^{p}(\mathbb{C})} \leq p^{*}-1$; the corresponding lower bound was already obtained by Lehto [16] in 1960s. So far, the best results in this direction is the inequality $\|B\|_{L^{p}(\mathbb{C}) \rightarrow L^{p}(\mathbb{C})} \leq 1.575\left(p^{*}-1\right)$ of Bañuelos and Janakiraman, and the estimate $\|B\|_{L^{p}(\mathbb{C}) \rightarrow L^{p}(\mathbb{C})} \leq 1.4(p-1)$ if $p \geq 1000$, due to Borichev, Janakiraman and Volberg [8]. Both these bounds were established with the use of probabilistic methods; more precisely, the proofs rest on certain martingale inequalities of Burkholder [9], [10] (see also Wang [20]) and their appropriate extensions.

In the study of T. Iwaniec' conjecture, it is natural to analyze the action of the Beurling-Ahlfors transform on some special classes of functions. The paper [7] of Baernstein and Montgomery-Smith contains some information in this direction for the class of the so-called stretch functions (see Sections 6 and 7 there). Another result of this type, proved by Bañuelos and Janakiraman $[5]$, is the estimate $\|B F\|_{L^{p}(\mathbb{C})} \leq\left(p^{*}-1\right)\|F\|_{L^{p}(\mathbb{C})}$, valid for $1<p \leq 2$ and $F$ being real-valued and radial, i.e., satisfying $F(z)=F(|z|)$ for all $z \in \mathbb{C}$. See also Bañuelos and Osȩkowski [6] for a different, probabilistic proof of this fact. On the other hand, we would like to point out that for $p>2$ and radial $F$ we have $\|B F\|_{L^{p}(\mathbb{C})} \leq \frac{2 p}{p-1}\|F\|_{L^{p}(\mathbb{C})}$ (see [6]) and the constant $2 p /(p-1)$ is better than $p^{*}-1=p-1$, at least for large $p$. Consult also the papers [5], [13] and [15] for other related facts.

We continue this line of research in the following direction. For $p=1$ and a radial function $F: \mathbb{C} \rightarrow \mathbb{C}$, the inequality $\|B F\|_{L^{1}(\mathbb{C})} \leq C_{1}\|F\|_{L^{1}(\mathbb{C})}$ does not hold in general with any finite constant $C_{1}$ : this can be verified directly by considering the examples of Lehto [16]. Thus it is natural to ask about a substitute for the $L^{1}$ estimate. One of the possible answers is to study appropriate weak-type bounds. In [5], Bañuelos and Janakiraman proved the following.

Theorem 1.1. Suppose that $F$ is a real-valued radial function. Then for any $\lambda>0$ we have

$$
\lambda|\{z \in \mathbb{C}:|B F(z)| \geq \lambda\}| \leq \frac{1}{\log 2}\|F\|_{L^{1}(\mathbb{C})}
$$

and the inequality is sharp.

The paper [6] contains the extension of this result. Among other things, it describes, for any $1 \leq p \leq 2$, the optimal constant $C_{p}$ such that for any 
radial function $F: \mathbb{C} \rightarrow \mathbb{C}$ we have the weak-type $(p, p)$ estimate

$$
\lambda^{p}|\{z \in \mathbb{C}:|B F(z)| \geq \lambda\}| \leq C_{p}^{p}\|F\|_{L^{p}(\mathbb{C})}^{p}, \quad \lambda>0 .
$$

The purpose of this paper is to study a different, LlogL inequality, which can also be regarded as a version of the $L^{p}$ estimate for $p=1$. In addition, we will consider the more general setting of vector-valued functions. Suppose that $\mathcal{H}$ is a separable Hilbert space over $\mathbb{R}$ (we may assume that $\mathcal{H}=\ell_{\mathbb{R}}^{2} \simeq$ $\left.\ell_{\mathbb{C}}^{2}\right)$, with norm $|\cdot|$ and scalar product $\langle\cdot, \cdot\rangle$. For any integrable function $f=\left(f_{1}, f_{2}, \ldots\right): \mathbb{C} \rightarrow \mathcal{H}$, we define $B f$ coordinatewise: that is, we set

$$
B f=\left(B f_{1}, B f_{2}, \ldots\right) \in \ell_{\mathbb{C}}^{2},
$$

or just observe that (1.1) makes sense for vector-valued functions. Throughout the paper, $\mathcal{B}(0, R)$ stands for the open ball of center 0 and radius $R$; furthermore, we introduce the function $\Psi:[0, \infty) \rightarrow[0, \infty)$ by the formula $\Psi(t)=(t+1) \log (t+1)-t$. We are ready to formulate our main result.

Theorem 1.2. Suppose that $F: \mathbb{C} \rightarrow \mathcal{H}$ is a radial function. Then for any $K>1$ and $R>0$ we have

$$
\frac{1}{|\mathcal{B}(0, R)|} \int_{\mathcal{B}(0, R)}|B F(z)| \mathrm{d} z \leq \frac{K}{|\mathcal{B}(0, R)|} \int_{\mathcal{B}(0, R)} \Psi(|F(z)|) \mathrm{d} z+L(K),
$$

where

$$
L(K)=\frac{K^{2} e^{K^{-1}}}{K+1}+\frac{2}{\left(K^{2}-1\right) e}-K .
$$

The constant $L(K)$ is the best possible for each $K$, even if we restrict ourselves to real-valued functions $F$. For $K \leq 1$ the inequality above does not hold with any finite $L(K)$.

This should be compared to the inequality

$$
\int_{A}|B F(z)| \mathrm{d} z \leq 2 K \int_{\mathbb{C}} \Psi(|F(z)|) \mathrm{d} z+\frac{|A|}{K-1}, \quad K>1,
$$

which holds true for all functions $F: \mathbb{C} \rightarrow \mathbb{C}$ and all Borel subsets $A$ of $\mathbb{C}$ (see [18]). Note that in the latter estimate the function $\Psi \circ|F|$ is integrated over all $\mathbb{C}$, and the multiplicative constant in front of the integral is at least 2. Furthermore, the term $|A| /(K-1)$ does not seem to be optimal here - we believe that the factor $(K-1)^{-1}$ can be improved (but we have not managed to show this). On the other hand, all the constants and restrictions in (1.2) are optimal and thus the inequality does give the full information on the interplay between the $L^{1}$-norm of $B F$ and the LlogL-norm of $F$.

A few words about the proof and the organization of the paper. We will deduce (1.2) from an appropriate sharp inequality for martingales, which is of independent interest. To establish this inequality, we will use an approach which can be regarded as an extension of Burkholder's method [10]: the proof will rest on the existence of a certain special function. This object is introduced and studied in Section 2 below and then, in Section 3, we exploit its properties to obtain the probabilistic version of (1.2). The final part of the paper is devoted to the proof of our main result, Theorem 1.2. 


\section{A special function}

Throughout this section, we assume that $K>1$ is a fixed number. Introduce the auxiliary function $\gamma:\left[0, e^{K^{-1}}-1\right] \rightarrow \mathbb{R}$, given by

$$
\gamma(x)=\frac{x+1}{K+1}+\frac{2(x+1)^{-K}}{K^{2}-1} .
$$

A straightforward calculation yields that

$$
(\gamma(x)-x)^{\prime}=-\frac{K}{K+1}-\frac{2 K(x+1)^{-K-1}}{K^{2}-1}<0, \quad x \in\left(0, e^{K^{-1}}-1\right),
$$

and thus the function $x \mapsto \gamma(x)-x$ is invertible. Let $H$ denote the inverse: then the domain of $H$ is precisely the interval $\left[x_{0},(K-1)^{-1}\right]$, where

$$
x_{0}=\gamma\left(e^{K^{-1}}-1\right)-e^{K^{-1}}+1=-\frac{K e^{K^{-1}}}{K+1}+\frac{2}{\left(K^{2}-1\right) e}+1>0
$$

(the latter inequality is proved in Lemma 2.3 below). Of course, the range of $H$ is equal to $\left[0, e^{K^{-1}}-1\right]$. Next, we check that $\gamma$ satisfies the differential equation

$$
\gamma^{\prime}(x)-1+\frac{K \gamma(x)}{x+1}=0, \quad x \in\left(0, e^{K^{-1}}-1\right)
$$

which implies that

$$
K H^{\prime}(x)(H(x)+x)=-H(x)-1 \quad \text { for } x \in\left(x_{0},(K-1)^{-1}\right) .
$$

The central role in the paper is played by the function $U:[0, \infty) \rightarrow \mathbb{R}$, given by

$$
U(x)= \begin{cases}L(K), & \text { if } x \leq x_{0}, \\ K(x-1) \log (H(x)+1)+K H(x), & \text { if } x_{0}<x \leq \frac{1}{K-1}, \\ (K-1) x-1-K(x+1) \log \left(\frac{K-1}{K}(x+1)\right), & \text { if } x>\frac{1}{K-1} .\end{cases}
$$

Let us list some properties of $U$.

\section{Lemma 2.1 .}

(i) The function $U$ is continuous on $[0, \infty)$ and of class $C^{1}$ in the interior of this interval.

(ii) The function $U$ is concave and nonincreasing.

Proof. (i): This is straightforward and left to the reader.

(ii): We have $U^{\prime}(0+)=0$, so it suffices to establish the concavity. The inequality $U^{\prime \prime}(x) \leq 0$ is evident for $x \in\left(0, x_{0}\right) \cup\left((K-1)^{-1}, \infty\right)$, while for $x \in\left(x_{0},(K-1)^{-1}\right)$ we derive that

$$
U^{\prime}(x)=K \log (H(x)+1)+\frac{K H^{\prime}(x)(H(x)+x)}{H(x)+1}=K \log (H(x)+1)-1,
$$

in view of (2.1). Thus, for all such $x$,

$$
U^{\prime \prime}(x)=\frac{K H^{\prime}(x)}{H(x)+1}=-\frac{1}{H(x)+x}<0
$$

and the proof is complete. 
For $x \in \mathcal{H}$, we define $x^{\prime}=x /|x|$ provided $x \neq 0$, and $x^{\prime}=0 \in \mathcal{H}$ otherwise. The main property of $U$ is described in the following statement.

Lemma 2.2. For any $x, h \in \mathcal{H}$ we have the estimate

$$
U(|x|)+U^{\prime}(|x|)\left\langle x^{\prime}, h\right\rangle \geq|h|-K \Psi(|x+h|) .
$$

To prove this property, we will need several technical lemmas.

Lemma 2.3. For any $K>1$ we have

$$
\begin{aligned}
e^{2 / K} & \leq 1+\frac{2}{K-1}, \\
\frac{K e^{K^{-1}}}{K+1} & \leq \frac{2}{\left(K^{2}-1\right) e}+1 .
\end{aligned}
$$

and

$$
\frac{e^{K^{-1}}}{K+1}+\frac{2}{\left(K^{2}-1\right) e} \leq \frac{1}{K-1} .
$$

Proof. The substitution $x=2 / K \in(0,2)$ transforms the first estimate into

$$
e^{x} \leq 1+\frac{x}{1-x / 2}=\frac{4}{2-x}-1 .
$$

It suffices to note that both sides become equal if we let $x \downarrow 0$ and

$$
\left(e^{x}-\frac{4}{2-x}\right)^{\prime}=\left(\frac{1}{e^{-x / 2}}\right)^{2}-\left(\frac{1}{1-x / 2}\right)^{2} \leq 0 .
$$

The bound (2.5) is proved similarly: we substitute $x=K^{-1} \in(0,1)$ and obtain the equivalent form

$$
(1-x) e^{x}-1+\left(1-2 e^{-1}\right) x^{2} \leq 0 .
$$

Both sides become equal in the limit $x \downarrow 0$ and, in addition, the derivative of the left-hand side is

$$
-x e^{x}+2\left(1-2 e^{-1}\right) x \leq-x+2\left(1-2 e^{-1}\right) x=x\left(1-4 e^{-1}\right) \leq 0 .
$$

Finally, (2.6) is shown analogously, with the use of the substitution $x=K^{-1}$. We leave the details to the reader.

Lemma 2.4. The function $F(t)=\Psi(\sqrt{t})$ is concave on $[0, \infty)$.

Proof. This is straightforward: for $t>0$ we have

$$
F^{\prime \prime}(t)=\frac{1}{4 t^{3 / 2}}\left[\frac{\sqrt{t}}{\sqrt{t}+1}-\log (\sqrt{t}+1)\right] \leq 0 .
$$

Lemma 2.5. For any $s \in\left[1, e^{K^{-1}}\right]$ we have

$$
s\left[s-2 \log s+2 K^{-1}(\gamma(s-1)-s+2)\right] \geq e^{2 / K} .
$$


Proof. Denote the left-hand side by $F(s)$. We easily derive that for $s \in$ $\left(1, e^{K^{-1}}\right)$ we have

$$
F^{\prime \prime}(s)=\frac{2}{s}[(K-1) \gamma(s-1)-1] .
$$

But this is nonpositive, equivalently, we have $\gamma(s) \leq(K-1)^{-1}$ for all $s$. To see this, observe that $\gamma$ is a convex function (which obvious from the very definition) and satisfies $\gamma(0)=(K-1)^{-1}$ and $\gamma\left(e^{K^{-1}}-1\right) \leq(K-1)^{-1}$, where the latter is precisely (2.6). So, we have proved that $F$ is a concave function, and thus it suffices to verify $(2.7)$ at the endpoints of the interval $\left[1, e^{K^{-1}}\right]$. For $s=1$ the bound reduces to (2.4), while for $s=e^{K^{-1}}$ it is equivalent to $(2.5)$.

We are ready to establish the main property of $U$.

Proof of Lemma 2.2. The proof is quite elaborate, so for the sake of convenience we have decided to split it into a few separate parts.

Step 1. The reduction to the real-valued case. By the continuity of both sides of (2.3), we may and do assume that $x \neq 0$. Fix $|x|$ and $|h|$. Observe that the estimate can be rewritten in the form $F(\langle x, h\rangle)$, where

$$
F(s)=U(x)+\frac{U^{\prime}(x)}{|x|} s-|h|+K \Psi\left(\sqrt{|x|^{2}+|h|^{2}+2 s}\right), \quad s \in[-|x||h|,|x||h|] .
$$

By Lemma 2.4, the function $F$ is concave, so it suffices to prove (2.3) for $x, h$ satisfying $\langle x, h\rangle \in\{-|x||h|,|x||h|\}$, i.e. in the case when these vectors are linearly dependent. That is to say, we may assume that $\mathcal{H}=\mathbb{R}$ and, replacing $x, h$ with $-x,-h$ if necessary, we may restrict ourselves to positive $x$.

Step 2. The case $x \leq x_{0}$. For such $x$, the inequality (2.3) reads

$$
L(K)+K \Psi(|x+h|)-|h| \geq 0 .
$$

We have $\Psi \geq 0$ and

$$
L(K)=x_{0}+K\left(e^{K^{-1}}-1\right)-1 \geq x_{0},
$$

so it suffices to show the inequality for $|h|>x_{0}$. Then

$$
L(K)+K \Psi(|x+h|)-|h| \geq L(K)+K \Psi\left(|h|-x_{0}\right)-|h|
$$

and it remains to observe that as a function of $|h| \in\left(x_{0}, \infty\right)$, the expression on the right is convex and vanishes, along with its derivative, at the point $|h|=\gamma\left(e^{K^{-1}}-1\right)$.

Step 3. The case $x_{0}<x \leq(K-1)^{-1}$. By virtue of $(2.2)$, the inequality (2.3) becomes

$$
\begin{aligned}
K(x-1) \log (H(x) & +1)+K H(x) \\
& +[K \log (H(x)+1)-1] h-|h|+K \Psi(|x+h|) \geq 0 .
\end{aligned}
$$

Fix $x$ and denote the left-hand side by $F(h)$. We easily check that $F$ is a convex function on $(-\infty, 0]$ and satisfies $F(-\gamma(H(x)))=F^{\prime}(-\gamma(H(x)))=0$; 
thus the above inequality holds true for nonpositive $h$. On the other hand, $F$ is also convex on $[0, \infty)$ and

$$
F^{\prime}(0+)=K \log [(H(x)+1)(x+1)]-2 .
$$

If this one-sided derivative is nonnegative, then automatically $F(h) \geq 0$ and we are done. On the other hand, if $F^{\prime}(0+)<0$, then a straightforward analysis of the derivative shows that $F$ attains its minimum at $h_{0}$ such that

$$
\log \left[(H(x)+1)\left(x+h_{0}+1\right)\right]=2 / K .
$$

This minimum equals

$$
\begin{aligned}
K\left(x+h_{0}+1\right) & \log \left[(H(x)+1)\left(x+h_{0}+1\right)\right] \\
& -2 K \log (H(x)+1)+K H(x)-2 h_{0}-K\left(x+h_{0}\right) \\
= & 2(x+1)-2 K \log (H(x)+1)+K H(x)-K\left(x+h_{0}\right),
\end{aligned}
$$

which is nonnegative. Indeed, if we write the inequality

$$
2(x+1)-2 K \log (H(x)+1)+K H(x) \geq K\left(x+h_{0}\right),
$$

divide throughout by $K$, add 1 to both sides and multiply by $H(x)+1$, we obtain the equivalent form

$$
(H(x)+1)\left[H(x)+1-2 \log (H(x)+1)+2 K^{-1}(x+1)\right] \geq e^{2 / K},
$$

by virtue of (2.8). It remains to substitute $s=H(x)+1 \in\left[1, e^{K^{-1}}\right]$ and make use of (2.7). Consequently, we have shown that $F$ is a nonnegative function on $\mathbb{R}$.

Step 4. The case $x>(K-1)^{-1}$. Here the reasoning is similar to that from the previous step. For $h \leq 0$, the inequality (2.3) can be rewritten in the form

$$
U(x)-K \log \left(\frac{K-1}{K}(x+1)\right) h+K \Psi(|x+h|) \geq 0 .
$$

Fix the number $x$. The left-hand side, as a function of $h$, is convex and vanishes, along with its derivative, for $h=-(x+1) / K$; this proves the estimate for all nonpositive $h$. If $h \geq 0$, the inequality reads

$$
F(h):=U(x)-\left[K \log \left(\frac{K-1}{K}(x+1)\right)+2\right] h+K \Psi(x+h) \geq 0 .
$$

The function $F$ is convex on $[0, \infty)$ and satisfies $F^{\prime}(0+)=K \log \frac{K}{K-1}-2$. If this derivative is nonnegative, then $F$ is positive on $[0, \infty)$ and we are done. On the other hand, if $K \log \frac{K}{K-1}<2$ (equivalently, $\frac{K}{K-1}<e^{2 / K}$ ), then it is easy to check that $F$ attains its minimum at $h_{0}$ satisfying

$$
\frac{x+h_{0}+1}{x+1}=\frac{K-1}{K} e^{2 / K} .
$$

Furthermore, the minimum equals $x+1-K h_{0} \geq 0$. To see the latter bound, we rewrite it in the form $h_{0} \leq(x+1) / K$, add $x+1$ to both sides and divide throughout by $x+1$. By $(2.9)$, the estimate becomes $\frac{K-1}{K} e^{2 / K} \leq \frac{K+1}{K}$, which has been established in (2.4). 
This completes the proof of (2.3).

\section{A martingale inequality}

As we have already announced in the first section, the heart of the matter lies in proving an appropriate martingale inequality. Let us start with introducing the necessary stochastic background. Suppose that $(\Omega, \mathcal{F}, \mathbb{P})$ is a probability space, filtered by $\left(\mathcal{F}_{t}\right)_{t \geq 0}$, a nondecreasing family of sub- $\sigma$-fields of $\mathcal{F}$, such that $\mathcal{F}_{0}$ contains all the events of probability 0 . Let $X=\left(X_{t}\right)_{t \geq 0}$ be an adapted continuous-time martingale, taking values in the Hilbert space $\mathcal{H}$. As usual, we assume that the trajectories of $X$ are right-continuous and have limits from the left. We shall also use the notation $\Delta X_{t}=X_{t}-X_{t}$ for the jump of $X$ at time $t \geq 0$ (we set $X_{0-}=X_{0}$, so that $\Delta X_{0}=0$ ). The symbol $[X, X]$ will stand for the quadratic covariance process of $X$ : that is, we put $[X, X]_{t}=\sum_{j=1}^{\infty}\left[X^{j}, X^{j}\right]_{t}$, where $X^{j}$ denotes the $j$-th coordinate of $X$. Here for real valued martingale $M,[M, M]$ is the usual square bracket of $M$, see e.g. Dellacherie and Meyer [11] for details.

The main result of this section is the following statement, which can be regarded as a probabilistic counterpart of Theorem 1.2.

Theorem 3.1. Suppose that $X$ is a bounded Hilbert-space-valued martingale and $\tau$ is a bounded stopping time. Then for any $K>1$ we have

$$
\mathbb{E}\left|\Delta X_{\tau}\right| \leq K \mathbb{E} \Psi\left(\left|X_{\tau}\right|\right)+L(K) .
$$

The constant $L(K)$ is the best for each $K$. If $K \leq 1$, then the above inequality does not hold with any finite $L(K)$.

Proof. Fix $K>1$. By Lebesgue's monotone convergence theorem, it suffices to show the estimate for finite-dimensional Hilbert-spaces: so, let us assume that $\mathcal{H}=\mathbb{R}^{d}$ for some $d \geq 1$. It follows from the second part of Lemma 2.1 that the function $x \mapsto U(|x|), x \in \mathbb{R}^{d}$, is concave; indeed, for any $x, y \in \mathbb{R}^{d}$ we have, by the triangle inequality,

$$
U(|\lambda x+(1-\lambda) y|) \geq U(\lambda|x|+(1-\lambda)|y|) \geq \lambda U(|x|)+(1-\lambda) U(|y|) .
$$

Next, let $g: \mathbb{R}^{d} \rightarrow[0, \infty)$ be a $C^{\infty}$ function, supported on the unit ball of $\mathbb{R}^{d}$ and satisfying $\int_{\mathbb{R}^{d}} g=1$. For any $\delta>0$, define $U^{\delta}: \mathbb{R}^{d} \rightarrow \mathbb{R}$ by the convolution

$$
U^{\delta}(x)=\int_{\mathbb{R}} U(|x+\delta r|) g(r) \mathrm{d} r .
$$

The function $U^{\delta}$ is of class $C^{\infty}$ and inherits the concavity from the function $x \mapsto U(|x|)$. Furthermore, since $U(s) \leq U(0)=L(K)$ for all $s>0$ (see Lemma 2.1 (ii)), we see that $U^{\delta}(x) \leq L(K)$ for all $x \in \mathbb{R}^{d}$. An application of Itô's formula yields

$$
U^{\delta}\left(X_{\tau}\right)=I_{0}+I_{1}+I_{2} / 2+I_{3}
$$


where

$$
\begin{aligned}
& I_{0}=U^{\delta}\left(X_{0}\right), \\
& I_{1}=\int_{0+}^{\tau} \nabla U^{\delta}\left(X_{s-}\right) \cdot \mathrm{d} X_{s}, \\
& I_{2}=\sum_{i, j=1}^{d} \int_{0+}^{\tau} \frac{\partial^{2}}{\partial x_{i} \partial x_{j}} U^{\delta}\left(X_{s-}\right) \mathrm{d}\left[X^{i}, X^{j}\right]_{s}^{c}, \\
& I_{3}=\sum_{0<s \leq \tau}\left[U^{\delta}\left(X_{s}\right)-U^{\delta}\left(X_{s-}\right)-\left\langle\nabla U^{\delta}\left(X_{s-}\right), \Delta X_{s}\right\rangle\right] .
\end{aligned}
$$

Let us analyze the terms $I_{0}-I_{3}$ separately. As we have observed above, $U^{\delta}$ does not exceed $L(K)$, so $I_{0} \leq L(K)$. Next, the process $\left(\nabla U^{\delta}\left(X_{s-}\right)\right)_{s>0}$ is bounded (since so is $X$ ), which implies that $\left(\int_{0+}^{t} \nabla U^{\delta}\left(X_{s-}\right) \cdot \mathrm{d} X_{s}\right)_{t \geq 0}$ is a mean-zero square-integrable martingale. Consequently, we have $\mathbb{E} I_{1}=0$. The term $I_{2}$ is nonpositive, which follows directly from the concavity of $U^{\delta}$. Finally, again by the concavity of $U^{\delta}$, we see that each summand appearing in $I_{3}$ is nonpositive; therefore we may write $I_{3} \leq U^{\delta}\left(X_{\tau}\right)-U^{\delta}\left(X_{\tau-}\right)-$ $\left\langle\nabla U^{\delta}\left(X_{\tau-}\right), \Delta X_{\tau}\right\rangle$. Plugging all these facts into (3.2) and taking expectation of both sides gives

$$
\mathbb{E}\left[U^{\delta}\left(X_{\tau-}\right)+\left\langle\nabla U^{\delta}\left(X_{\tau-}\right), \Delta X_{\tau}\right\rangle\right] \leq L(K) .
$$

Now we let $\delta \rightarrow 0$ and use Lebesgue's dominated convergence theorem to obtain

$$
\mathbb{E}\left[U\left(\left|X_{\tau-}\right|\right)+U^{\prime}\left(\left|X_{\tau-}\right|\right)\left\langle X_{\tau-}^{\prime}, \Delta X_{\tau}\right\rangle\right] \leq L(K) .
$$

In consequence, by Lemma 2.2, we obtain

$$
\mathbb{E}\left|\Delta X_{\tau}\right|-K \mathbb{E} \Psi\left(\left|X_{\tau}\right|\right) \leq L(K),
$$

which is the claim. The sharpness of this estimate will be clear from the optimality of $L(K)$ in (1.2): see the next section and a remark at the end of it.

\section{Proof of Theorem 1.2}

\subsection{Proof of (1.2)}

We start from describing the action of the Beurling-Ahlfors operator on the class of radial functions. For any square-integrable $f:[0, \infty) \rightarrow \mathbb{R}$, let $F$ be the associated radial function, given by $F(z)=f\left(|z|^{2}\right), z \in \mathbb{C}$. Then, as proved in [5], we have

$$
B F(z)=\frac{\bar{z}^{2}}{|z|^{2}} \Lambda f\left(|z|^{2}\right),
$$

where the operator $\Lambda$ is defined by

$$
\Lambda f(u)=\frac{1}{u} \int_{0}^{u} f(v) \mathrm{d} v-f(u) .
$$


Of course, the above two formulas make sense if $f$ takes values in $\mathcal{H}$, simply by applying (4.1), (4.2) coordinatewise. Next, let $f:[0, \infty) \rightarrow \mathcal{H}$ be a bounded function. Fix $R>0$, put $M=R^{2}$ and consider the probability space

$$
(\Omega, \mathcal{F}, \mathbb{P})=([0, M], \mathfrak{B}([0, M]),|\cdot| / M),
$$

where $\mathfrak{B}([0, M])$ denotes the family of all Borel subsets of $[0, M]$ and $|\cdot|$ stands for the Lebesgue's measure. For any $t \in[0, M]$, let $\mathcal{F}_{t}$ be the smallest complete $\sigma$-field which contains the interval $[0, M-t]$ and all Borel subsets of $[M-t, M]$; for $t>M$, we put $\mathcal{F}_{t}=\mathcal{F}$. Obviously, $\left(\mathcal{F}_{t}\right)_{t \geq 0}$ is a filtration, $f$ can be regarded as an integrable random variable and thus the process

$$
X=\left(X_{t}\right)_{t \geq 0}=\left(\mathbb{E}\left(f \mid \mathcal{F}_{t}\right)\right)_{t \geq 0}
$$

is a bounded martingale. It is easy to see that for almost all $\omega \in \Omega$,

$$
X_{t}(\omega)= \begin{cases}f(\omega), & \text { if } t \geq M-\omega, \\ \frac{1}{M-t} \int_{0}^{M-t} f(s) \mathrm{d} s, & \text { if } t<M-\omega .\end{cases}
$$

Furthermore, the random variable $\tau(\omega)=M-\omega$ is an adapted, bounded stopping time: indeed, for any $t \geq 0$ we have $\{\tau \leq t\}=[(M-t) \vee 0, M] \in \mathcal{F}_{t}$. Therefore, by virtue of Theorem 3.1 , for any fixed $K>1$ we have

$$
\begin{aligned}
\frac{1}{M} \int_{0}^{M}|\Lambda f(x)| \mathrm{d} x=\mathbb{E}\left|\Delta X_{\tau}\right| & \leq K \mathbb{E} \Psi\left(\left|X_{\tau}\right|\right)+L(K) \\
& =\frac{K}{M} \int_{0}^{M} \Psi(|f(x)|) \mathrm{d} x+L(K) .
\end{aligned}
$$

Now apply (4.1) and recall that $M=R^{2}$ to get

$$
\begin{aligned}
\int_{\mathcal{B}(0, R)}|B F(z)| \mathrm{d} z & =\int_{\mathcal{B}(0, R)}\left|\Lambda f\left(|z|^{2}\right)\right| \mathrm{d} z \\
& =\int_{0}^{R}\left|\Lambda f\left(r^{2}\right)\right| 2 \pi r \mathrm{~d} r \\
& =\pi \int_{0}^{R^{2}}|\Lambda f(r)| \mathrm{d} r \\
& \leq \pi K \int_{0}^{R^{2}} \Psi(|f(r)|) \mathrm{d} r+\pi R^{2} L(K) \\
& =K \int_{\mathcal{B}(0, R)} \Psi(|F(z)|) \mathrm{d} z+L(K) \cdot|\mathcal{B}(0, R)| .
\end{aligned}
$$

This yields the logarithmic estimate for bounded functions. The general case follows immediately by a standard approximation.

\subsection{Sharpness}

We exhibit an appropriate example. For a fixed $K>1$, let $f:[0,1] \rightarrow \mathbb{R}$ be given by

$$
f(t)= \begin{cases}(e t)^{-1 / K}-1, & \text { if } t \in\left(0, e^{-1}\right], \\ 1-(e t)^{1 / K}, & \text { if } t \in\left(e^{-1}, 1\right] .\end{cases}
$$


A direct computation shows that

$$
\Lambda f(t)= \begin{cases}(e t)^{-1 / K}(K-1)^{-1}, & \text { if } t \in\left(0, e^{-1}\right] \\ \frac{2}{\left(K^{2}-1\right) e t}+(e t)^{1 / K}(K+1)^{-1}, & \text { if } t \in\left(e^{-1}, 1\right] .\end{cases}
$$

Now lengthy, but straightforward calculations lead to

$$
\int_{0}^{1}|\Lambda f(t)| \mathrm{d} t=\frac{4 K^{2}}{\left(K^{2}-1\right)^{2} e}+\frac{2}{\left(K^{2}-1\right) e}+\frac{K e^{1 / K}}{(K+1)^{2}}
$$

and

$$
\begin{aligned}
K \int_{0}^{1} \Psi(|f(t)|) \mathrm{d} t= & \frac{2 K^{2}\left(K^{2}+1\right)}{\left(K^{2}-1\right)^{2} e}+\frac{K}{(K+1)^{2}} e^{1 / K} \\
& -K\left(\frac{2 K}{\left(K^{2}-1\right) e}-1+\frac{K}{K+1} e^{1 / K}\right) .
\end{aligned}
$$

Combining these two equalities, we obtain

$$
\int_{0}^{1}|\Lambda f(t)| \mathrm{d} t-K \int_{0}^{1} \Psi(|f(t)|) \mathrm{d} t=L(K) .
$$

Therefore, if we let $F: \mathbb{C} \rightarrow \mathbb{R}$ be given by $F(z)=f\left(|z|^{2}\right) 1_{\{|z| \leq 1\}}$, then the calculation presented in (4.3) yields

$$
\frac{1}{|\mathcal{B}(0,1)|} \int_{\mathcal{B}(0,1)}|B F(z)| \mathrm{d} z=\frac{K}{|\mathcal{B}(0,1)|} \int_{\mathcal{B}(0,1)} \Psi(|F(z)|) \mathrm{d} z+L(K) .
$$

This implies that $L(K)$ is optimal for any $K>1$. Finally, the inequality (1.2) does not hold with any $L(K)<\infty$ when $K \leq 1$. This is the direct consequence of the fact that $L(K)$ explodes as $K$ decreases to 1 .

Remark 4.1. Of course, the reasoning above implies that the constant $L(K)$ in (3.1) is also sharp (and that the inequality does not hold for $K \leq 1$ with any finite $L(K))$. Indeed, if (3.1) were valid with a better constant, this would yield the corresponding improvement of (1.2) which, as we have just shown, is impossible.

\section{Acknowledgments}

I would like to thank the anonymous referee for the careful reading of the first version of the paper.

\section{References}

[1] K. Astala, Area distortion of quasiconformal mappings, Acta Math. 173 (no. 1) (1994), 37-60.

[2] K. Astala, T. Iwaniec and G. Martin, Elliptic partial differential equations and quasiconformal mappings in the plane, Princeton University Press, Princeton, NJ, 2009.

[3] R. Bañuelos, The foundational inequalities of D. L. Burkholder and some of their ramifications, Illinois J. Math. (no. 3) 54 (2010), 789-868. 
[4] R. Bañuelos and P. Janakiraman, $L_{p}$-bounds for the Beurling-Ahlfors transform, Trans. Amer. Math. Soc. 360 (2008), 3603-3612.

[5] R. Bañuelos and P. Janakiraman, On the weak-type constant of the BeurlingAhlfors Transform, Michigan Math. J. 58 (2009), 239-257.

[6] R. Bañuelos and A. Osȩkowski, Sharp inequalities for the Beurling-Ahlfors transform on radial functions, Duke Math. J., to appear.

[7] A. Baernstein II and S. J. Montgomery-Smith, Some conjectures about integral means of $\partial f$ and $\bar{\partial} f$, in: Complex Analysis and Differential Equations (Uppsala, Sweden, 1997), Ch. Kiselman ed., Acta. Univ. Upsaliensis Univ. C Organ. Hist. 64 (1999), Uppsala Univ. Press, Uppsala, Sweden, 92-109.

[8] A. Borichev, P. Janakiraman and A. Volberg, Subordination by conformal martingales in $L^{p}$ and zeros of Laguerre polynomials, preprint.

[9] D. L. Burkholder, Boundary value problems and sharp inequalities for martingale transforms, Ann. Probab. 12 (1984), 647-702.

[10] D. L. Burkholder, Explorations in martingale theory and its applications, École d'Ete de Probabilités de Saint-Flour XIX - 1989, Lecture Notes in Math. 1464, Springer, Berlin, 1991, 1-66.

[11] C. Dellacherie and P.-A. Meyer, Probabilities and potential B: Theory of martingales, North Holland, Amsterdam, 1982.

[12] F. W. Gehring, E. Reich, Area distortion under quasiconformal mappings, Ann. Acad. Sci. Fenn. Ser. A I 388 (1966) pp. 1- 15.

[13] A. Hinkkanen, On the norm of the Beurling-Ahlfors transformation, preprint.

[14] T. Iwaniec, Extremal inequalities in Sobolev spaces and quasiconformal mappings, Z. Anal. Anwendungen 1 (1982), 1-16.

[15] T. Iwaniec, Hilbert transform in the complex plane and area inequalities for certain quadratic differentials, Michigan Math. J. 34 (no. 3) (1987), 407-434.

[16] O. Lehto, Remarks on the integrability of the derivatives of quasiconformal mappings, Ann. Acad. Sci. Fenn. Series A I Math. 371 (1965), 3-8.

[17] O. Lehto and K. I. Virtanen, Quasiconformal mappings in the plane, Second edition, Springer-Verlag, New York-Heidelberg, 1973.

[18] A. Osȩkowski, Logarithmic inequalities for Fourier multipliers, Math. Z., to appear.

[19] E. M. Stein, Singular integrals and Differentiability Properties of Functions, Princeton University Press, Princeton, 1970.

[20] G. Wang, Differential subordination and strong differential subordination for continuous-time martingales and related sharp inequalities, Ann. Probab. 23 (no. 2) (1995), 522-551.

Adam Osȩkowski

Faculty of Mathematics, Informatics and Mechanics, University of Warsaw

Banacha 2, 02-097 Warsaw

Poland

e-mail: ados@mimuw.edu.pl

Received: September 28, 2012.

Accepted: December 10, 2012.

Open Access This article is distributed under the terms of the Creative Commons Attribution License which permits any use, distribution, and reproduction in any medium, provided the original author(s) and source are credited. 